\title{
Trabajo y polisemia
}

\author{
Mercedes Blanco
}

$\mathrm{P}$ OLISEMIa: este calificativo, que denota la pluralidad de significados de una palabra, pareciera estar casi reservado para aquellos términos que, de entrada, pudieran causar la impresión de ser poco precisos, ambiguos y hasta confusos. Es por ello que tal vez para muchas personas no especializadas en el tema sería un tanto difícil pensar que el vocablo "trabajo" y el verbo "trabajar" requieran de extensas discusiones para llegar a definiciones y precisiones por cuanto, de hecho, conforma uno de los ejes rectores en la construcción del curso de vida de hombres y mujeres en todo el mundo y, por lo tanto, nos remite a una situación o actividad sumamente cercana. Debiera ser, entonces, fácil responder a preguntas tan aparentemente simples como ¿qué se entiende por trabajar? o ¿qué significa "tener un trabajo"? No importa mucho cómo se verbalicen estas interrogantes, el punto central que es necesario destacar es la enorme discusión conceptual, teórica, académica y también política, administrativa e institucional que preguntas "tan simples" han suscitado. Baste recordar a los clásicos, como Adam Smith y Karl Marx, pensando en el trabajo como "un proceso mediante el cual el hombre transforma la naturaleza”, y muchas décadas después a las

MERCEDES BLANCO: CIESAS -D.F

Desacatos, núm. 11, primavera 2003, pp. 97-102. instancias gubernamentales de todo el mundo que captan y generan información hablando casi coloquialmente de "la PEA", esa "población económicamente activa" que ha ido variando en su concepción pero que básicamente busca captar a aquellas personas que "realizan una actividad económica", o sea, que producen bienes y servicios que van al mercado (otro concepto que, aún en la actualidad, provoca discusiones en cuanto a sus especificaciones y dinámica) para ser intercambiados, y por lo cual se reciben diferentes tipos de pago - fundamentalmente, dinero o pago en especie.

No es la intención ofrecer aquí una especie de "glosario de términos", sino simplemente señalar la discusión aún vigente para precisar una serie de conceptos relacionados con el amplio mundo del trabajo. Dichas discusiones incluyen, no sólo aquellos términos relacionados con la actividad de trabajar, estar ocupado/a, estar empleado/a, ser económicamente activo/a, etc., sino sus contrapartes que, de alguna manera, pueden concentrarse en los álgidos debates sobre qué significa y cómo captar, por ejemplo, el desempleo. ${ }^{1}$

La necesidad de contar con definiciones (más allá de la discusión de si éstas encasillan o, por el contrario, aclaran

\footnotetext{
${ }^{1}$ En el mundo anglosajón connotados especialistas internacionales siguen dedicando parte de sus reflexiones a aclarar las distinciones y diferencias que existen, por ejemplo, entre los vocablos que en inglés forman un trío conceptual: "work, labour, employment" (Standing, 1999).
} 
y son útiles) nos remite al terreno de lo conceptual y de ahí, a su vez, al teórico; ámbitos con los que cualquier investigación en ciencias sociales tendría que tener contacto; de esto se seguiría la — para muchos/as investigadores/as - indispensable relación entre lo teórico y lo empírico, dependiendo de la posición epistemológica que se asuma. En este espacio no es posible entrar a la muy conocida discusión en torno a la manera de llevar a cabo una investigación y generar conocimiento lo cual lleva al debate sobre si existe o no un solo "método científico", si éste necesariamente está inscrito en el paradigma positivista o neopositivista, si sólo este tipo de método es válido para alcanzar la meta de obtener conocimiento y de si en realidad es útil y aplicable a todo tipo de ciencias (desde las llamadas "duras" hasta las humanidades). Tampoco es posible dar cabida a las réplicas que se ubican como un polo enemigo y que pretenden descalificar absolutamente la necesidad de contar con algún tipo de método o metodología que guíe la investigación. ${ }^{2}$ Con la intención de sobrepasar esta discusión dicotómica, a finales de los años noventa cobró nuevo impulso la propuesmixta" que, como su nombre lo indica, busca no sólo la complementariedad y la combinación de enfoques y niveles sino incluso se ubica en un continuum de métodos (Newman y Benz, 1998; Tashakkori y Teddlie, 1998).

Los estudiosos del mercado y la fuerza de trabajo también han transitado por una variedad de caminos y opciones teórico-metodológicas que, como en muchos otros temas de investigación, han pasado a lo largo de los años de las propuestas un tanto simplistas, mecánicas y homogeneizantes a la consideración de la diversidad y la complejidad, a lo interdisciplinario y a lo "multi", o sea, lo multidimensional, lo multifactorial, lo multicausal, los multimétodos, los multiniveles, lo multicutural, etc., etc. Los artículos que se incluyen en este volumen son un ejemplo de esta diversidad ya que abarcan desde

2 Recordemos que la acepción tal vez más sencilla de la palabra metodología — proveniente de su propia etimología griega — la concibe como un camino o una manera o, dicho tal vez más propiamente, como un procedimiento para llegar al objetivo de conocer, de indagar, sobre un cierto fenómeno.

uno de los ámbitos laborales más formales y estructurados, como el que se da en las grandes empresas donde Guadalupe Serna estudia a mujeres ejecutivas, hasta el polo opuesto que representa una de las actividades con condiciones más precarias, vulnerables y de explotación como lo es el ejercer la prostitución callejera en Oaxaca, que es uno de los estados de la República mexicana que se caracteriza por sus fuertes rezagos socioeconómicos.

Estos dos artículos, así como aquél que se ocupa de la salud de las telefonistas, firmado por Josefina Ramírez, parecieran, en primer lugar, atender a la propuesta de una agenda general de investigación donde se hace la propuesta de estudiar las especificidades, demanda totalmente vigente para la comprensión de ese amplio mundo del trabajo cada vez más diverso, cambiante y fragmentado. Cada uno de los textos contiene elementos que fácilmente pueden llevar a reflexionar sobre grandes temas ampliamente conocidos, tales como la división del trabajo (social y sexual), la vinculación existente entre trabajo doméstico y extra-doméstico (esferas reproductiva-productiva) o la famosa doble jornada, entre muchos otros, sin embargo, a continuación se hará referencia sólo a algunas temáticas más puntuales que surgieron de la lectura de los textos que se incluyen en este volumen de la revista Desacatos.

Guadalupe Serna elige estudiar un grupo ocupacional ciertamente poco investigado en México lo cual, de entrada, le confiere un carácter novedoso y valioso a esta investigación, sin embargo, el haber sido poco estudiado no significa que este segmento ocupacional haya sido ignorado tanto en los estudios de corte cualitativo como en la cifras que la autora busca rescatar de diferentes bases de datos. Como le sucede muchas veces a los/as investigadores/as cuando se acercan a las fuentes estadísticas, se encuentran con una serie de limitaciones, e incluso carencias, del tipo de información que ofrecen censos y encuestas. Es por ello que las evaluaciones de las fuentes de datos y los comentarios críticos en torno a éstas siempre son no sólo bienvenidas sino incluso necesarias al hacer uso de y presentar en los textos información estadística.

Este tipo de consideraciones hubiera sido ciertamente pertinente en el artículo sobre las mujeres ejecutivas pues está ausente, por ejemplo, el hacer aunque sea una breve 


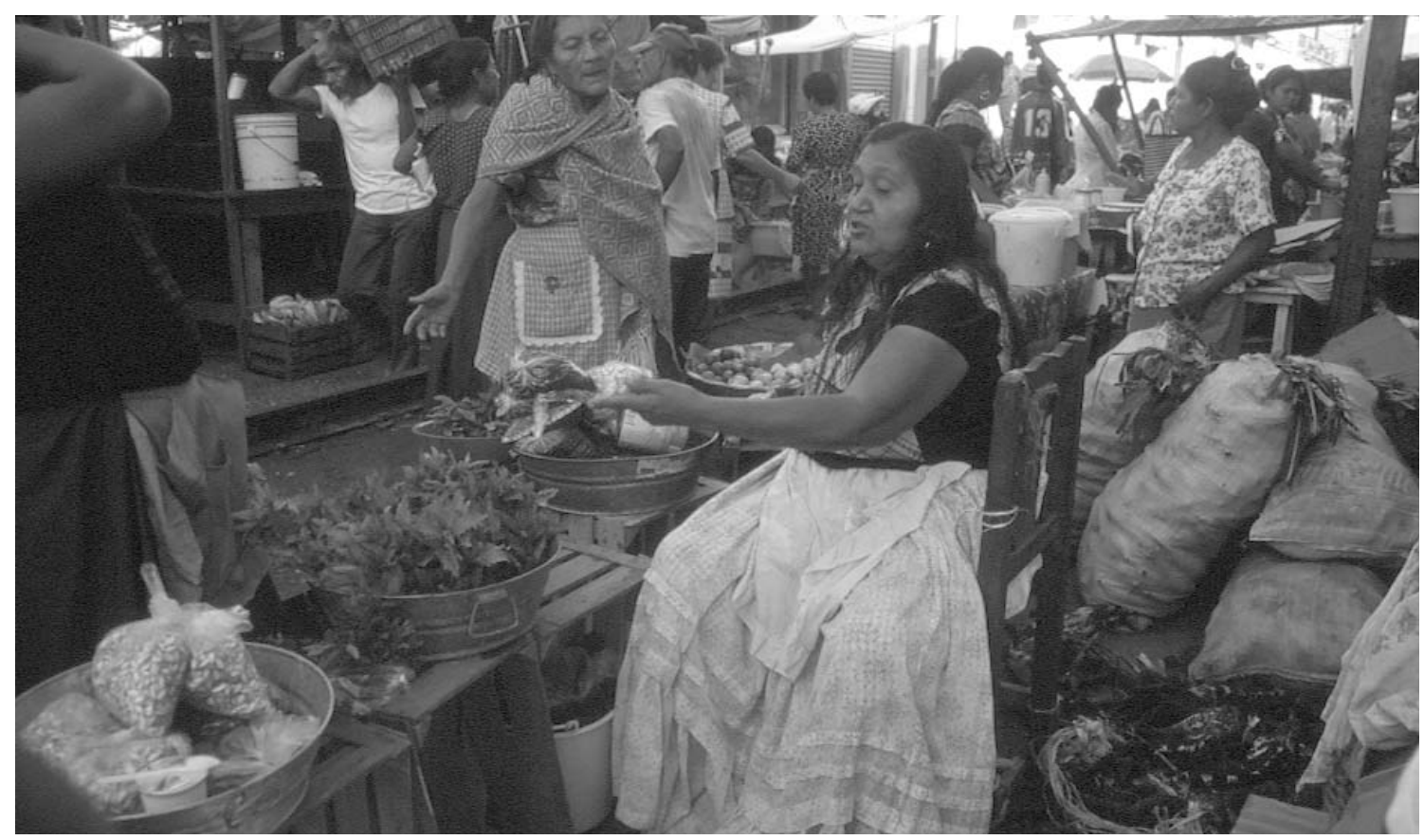

De la serie: Mercados hecho a mano, 2002 / Roxana Acevedo

referencia a una discusión que desde hace años se ha dado entre los/as especialistas en la generación y el análisis de información estadística específicamente referida al ámbito laboral, se trata de aquella que analiza las ventajas y desventajas que presentan los Censos Generales de Población frente a las encuestas diseñadas especialmente para captar una variedad de elementos sobre el mundo del trabajo (entre otras, pero principalmente, en el caso de México, la Encuesta Nacional de Empleo Urbano y la Encuesta Nacional de Empleo).

Una de las vertientes relevantes es precisamente aquella que tiene que ver con la medición del trabajo femenino, preocupación que podría decirse que surge de uno de los postulados u objetivos de la perspectiva de género que es aquel de "hacer visible lo invisible". En este proceso hay que reconocer el esfuerzo llevado a cabo por algunos grupos de investigadoras/es que han impulsado la producción de estadísticas con enfoque de género en México ya que están convencidas/os del papel decisivo que juega este tipo de información en "hacer visible la desigualdad de género" (INEGI, 1995; Inmujeres et al.,
2001). Por lo que toca al ámbito laboral, lo que ahora tal vez pueda sonar ya muy trillado sigue siendo, sin embargo, un objetivo no alcanzado a plenitud, o sea, la necesidad de sacar a la luz y valorar (en cualquier sentido, no sólo, por ejemplo, en una lectura maniquea de trabajo productivo vs improductivo) toda una gama de actividades llevadas a cabo casi exclusivamente por las mujeres y que van, como ahora es ya consabido, desde el trabajo doméstico hasta el "trabajo familiar no remunerado" que captan las fuentes de datos estadísticos. En este sentido, la discusión ha ido por el lado de las discrepancias que a veces se encuentran entre censos y encuestas, donde las segundas han buscado rescatar la mayor variedad posible de actividades desempeñadas por las mujeres, aún dentro del propio trabajo considerado como extradoméstico (o en la esfera productiva) pero sin limitarse a la idea de producción para el mercado bajo formas asalariadas. Algunos/as investigadores/as han expresado el temor de que las encuestas de empleo, en su esfuerzo por hacer visible la gama de actividades desempeñadas por las mujeres, pudiesen registrar como económicamente activas a 


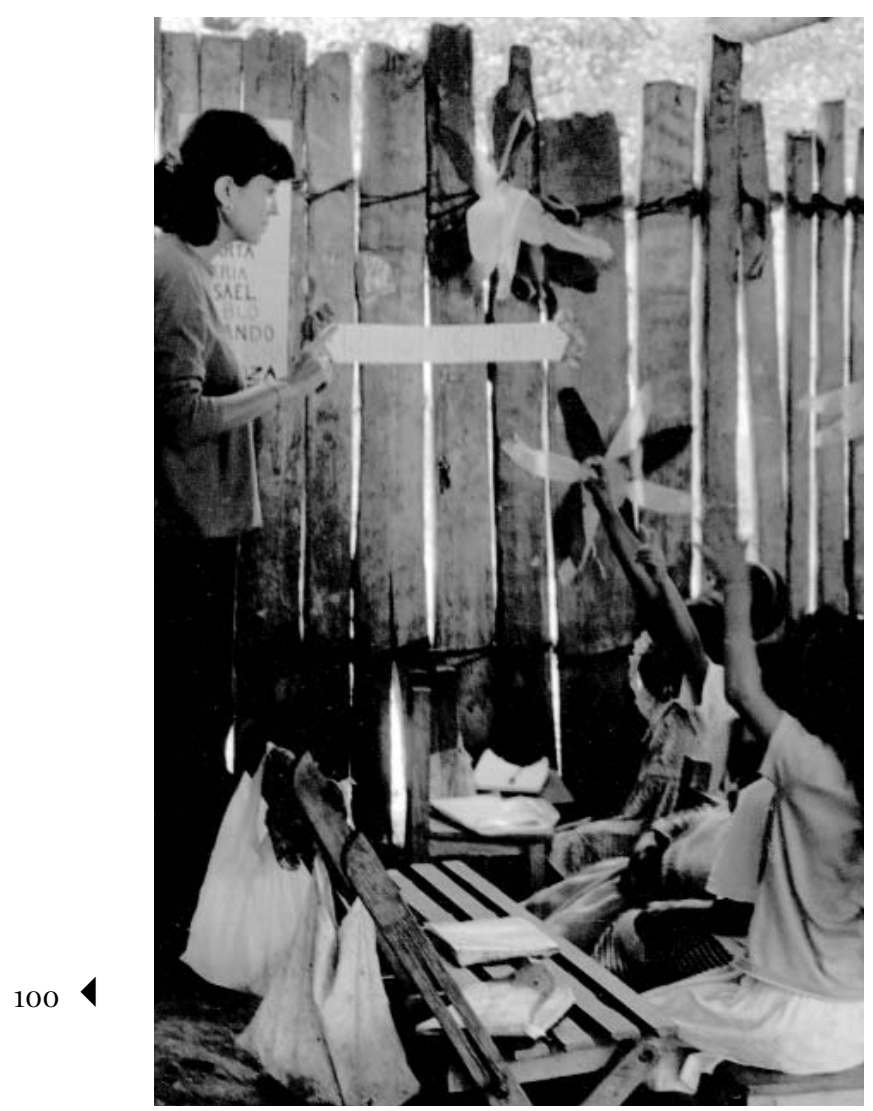

De la serie: Alto, mujeres trabajando, 2003 / Roxana Acevedo

personas que realizan tareas exclusivamente para el consumo privado, en vez de trabajos destinados a la producción y el intercambio; así, el debate continua pero lo que es un hecho es que la reflexión sobre el concepto de trabajo ha resultado muy importante en el avance de la discusión en torno a la subordinación y "empoderamiento" de las mujeres (García, Blanco y Pacheco, 1999).

Si en algún tipo de situación está dirigido el esfuerzo por producir información con perspectiva de género es precisamente la que da cuenta Jayne Howell, o sea, actividades de las que incluso algunos dudan que puedan ser calificadas como trabajo; éste es el caso de la prostitución. Es claro que no sólo ejercer la prostitución, sino las condiciones que este texto nos reseña para las calles de la ciudad de Oaxaca, se ubica en el extremo de las condiciones de precariedad. El tener de primera mano y de viva voz la expresión de situaciones de este tipo resulta muy ilustrativo pero esto no implica omitir el señalamiento de que hay una serie de puntos que ameritarían ser tratados con más cuidado.

Por ejemplo, es claro que la investigación de Howell, al igual que las de Serna y Ramírez, se ubican como estudios eminentemente de corte cualitativo y, sin embargo, se echa mano de "estudios estadísticos", como los califica Howell, para contextualizar a sus propios sujetos, o dicho más llanamente, a sus entrevistadas. Esta modalidad no sólo es cada vez más usual sino también pertinente y podríamos decir que hasta "correcta" pero, nuevamente, habría que hacer una mínima evaluación de las fuentes utilizadas pues se sabe que si algo es difícil de contabilizar es precisamente el famoso "sector informal", ya no digamos actividades que rayan en la ilegalidad o la clandestinidad (aunque, en estricto sentido, prostituirse no sea un delito en Oaxaca, como lo indica la autora). Es por ello que hablar de "trabajadoras sexuales mexicanas" puede ser inapropiado ya que, probablemente, los estudios citados se refieran a universos mucho más acotados. En este mismo tenor, la autora hacer referencia a "su muestra" cuando en realidad está lejos de serlo, por ello, sería conveniente no utilizar términos más propios de la estadística ya que muchas veces su aplicación a estudios cualitativos resulta un tanto forzada.

Después de haber leído como dos polos de un espectro los textos dedicados a las altas ejecutivas y a las prostitutas en condiciones muy precarias, la referencia a un grupo de trabajadoras asalariadas, además en un ámbito laboral altamente formalizado y también conocido por sus luchas sindicales, como lo es el de los servicios telefónicos, nos sugiere una variedad de grandes líneas de reflexión pero, atendiendo al interés central que expresa la autora en su texto, se abordarán sólo algunos puntos de un tema sumamente amplio y complejo: el del papel de la tecnología en el mundo del trabajo.

En realidad, la propia autora acota este vasto tema al centrarse en la relación entre introducción de nueva tecnología y salud, más específicamente, ubicada como "la problemática del estrés". También reconoce que hablar de causas y efectos del estrés no es nada fácil y, habría que agregar, pues rápidamente se podría caer 
en explicaciones simplistas o mecánicas. Aunque Josefina Ramírez está consciente de que esta relación está mediada por múltiples elementos, la autora destaca lo que llama "la experiencia personal" para llegar a la propuesta de la vivencia del estrés como expresión de resistencia entre las trabajadoras expuestas a la adopción de cambios tecnológicos.

Resulta una hipótesis muy interesante, sin embargo, parece estar un tanto desvinculada de un contexto más amplio, precisamente el macroestructural, que al parecer sí sería del interés de la autora incluir en su análisis, como lo expresa al inicio de su texto. Es decir, desde el planteamiento del problema se contempla la relación "macro-micro" al vincular al proceso de modernización tecnológica con el padecimiento del estrés, pero en el afán de rescatar a los sujetos — a las vidas individuales de las trabajadoras - se pierde un tanto el nivel macro aunque, queda claro, no es el ámbito que la autora quiere rescatar en particular pero sí, tal vez, la relación entre ambas dimensiones. Ciertamente no es una tarea fácil tratar de articular en la investigación y en el análisis los famosos niveles "micro-macro", de hecho sigue representando un reto teórico-metodológico para las ciencias sociales, claramente presente por lo menos desde la década de 1980 (Knorr-Cetina y Cicourel, 1981; Alexander et al., 1987), sin embargo, vale la pena correr el riesgo de experimentar con soluciones a dicho problema.

Volviendo a la problemática de los efectos que puede acarrear en la salud de los/as trabajadores/as la modernización de los procesos de trabajo, entre otros, uno de los factores importantes que están presentes en ese complejo síndrome que representa el estrés, en este caso, de las telefonistas, es el temor de estas mujeres a ser desplazadas por la introducción de nuevas tecnologías y, por ende, a perder el empleo. Los grandes organismos internacionales dedicados a abordar una variedad de temáticas en torno al trabajo, en su estudio sobre las tendencias mundiales del empleo y, más específicamente, a propósito del impacto de las nuevas tecnologías de la información y la comunicación en la vida laboral, afirman que este fenómeno ciertamente vuelve obsoletas e innecesarias cierto tipo de actividades y ocupaciones - lo cual podría traducirse, por supuesto, en pérdida de empleo- pero es igualmente cierto que se crean nuevas tareas, oficios, especialidades y puestos de trabajo (OIT, 2002).

No se trata de minimizar la realidad de la creciente dificultad de las economías del mundo para crear empleos con buenas condiciones de trabajo, pero sí parece necesario introducir ciertos matices en torno a la amenaza que representa la modernización tecnológica. Aunque suene a "cliché", ésta provoca a la vez crisis y oportunidad, situación que en el caso de las tecnologías de la información y la comunicación atañe directamente a las mujeres pues parte de las nuevas actividades requeridas por la tecnología se traducen en trabajos que pueden desempeñar las mujeres. Entre otros casos históricos, se puede recordar como la introducción de la máquina de escribir, desde fines del siglo XIX pero, sobre todo, a principios del XX, no sólo modificó en buena medida el funcionamiento de las oficinas sino, además, provocó a la larga la feminización de un mercado de trabajo que había sido — casi como todos- eminentemente masculino (Davies, 1982). También hay que enfatizar, como es muy conocido, que estos procesos están fuertemente caracterizados por la segregación ocupacional por sexo con todas las desventajas que conlleva para las trabajadoras.

Finalmente, el artículo dedicado a las ONG's se ubica en un nivel de análisis muy diferente al de los otros textos. Desde esa plataforma macroestructural los movimientos sociales y las organizaciones no gubernamentales de mujeres son objeto de lo que parece una severa crítica ya que, según afirma jules Falquet, algunos de los grandes organismos internacionales, tales como la ONU y el FMI, han propiciado la burocratización y la competencia entre movimientos y grupos interesados en promover diversos aspectos relacionados con el bienestar de las mujeres en todo el mundo. Falquet retoma del "análisis feminista autónomo latinoamericano y caribeño" una especie de diagnóstico por demás contundente respecto a esta situación: "la despolitización del movimiento y su pérdida de autonomía conceptual y organizativa $-\mathrm{y}$ por tanto de radicalidad y de potencialidad transformadora”. A continuación el autor ofrece algunos ejemplos que buscan demostrar que los programas y políticas llevadas a cabo por los organismos internacionales antes citados son "particularmente perjudiciales para las mujeres". 
Seguramente lo primero que hay que decir es que sería deseable contar con la opinión especializada de estudiosos/as que se han dedicado a realizar análisis minuciosos del fenómeno de la proliferación de las ONG's pero, a falta de ello en este momento, el comentario tal vez más obvio y/o general es que sería necesario matizar, relativizar, no homogeneizar, a pesar de que el mismo autor dice estar consciente de que tanto organismos internacionales como ONG's no conforman bloques monolíticos.

Es sin duda incuestionable que los organismos internacionales, como prácticamente cualquier grupo o institución, responden a intereses específicos y, por ende, seguramente habrá segmentos beneficiados y otros afectados por sus acciones y políticas, pero los análisis o reflexiones ubicados en un alto grado de generalidad - como es el caso de este texto- parecieran requerir de una mayor variedad de ejemplos que pudieran ilustrar las varias facetas y efectos, aunque sean contradictorios, para finalmente evaluar - por qué no - las ganancias y las pérdidas. Sin reivindicar, de ninguna manera, una vertiente asistencialista de degradación y manipulación de y tampoco sin hacer a un lado la crítica más severa que merece todo tipo de instituciones nacionales e internacionales, la impresión que queda al terminar de leer este artículo es la de que no hay absolutamente nada rescatable - para las mujeres concretas, las de "carne y hueso"- de la institucionalización de una variedad de procesos que conllevan las acciones de dichos organismos internacionales.

Para terminar, y como colofón a los comentarios vertidos en relación con los cuatro textos que comprende esta sección de la revista Desacatos, parece pertinente apuntar aquella posición que manifiesta que

...la investigación no puede restringirse a explicar los obstáculos, la pasividad y la discriminación que enfrenta la mujer en su incorporación plena a la sociedad. En primer lugar, porque un trabajo orientado por perspectivas de esta índole se limitaría a medir con más o menos precisión lo que sabemos de antemano, evitando así la construcción teórica y, en segundo lugar, porque ello oculta que, en las últimas décadas, una de las características más destacadas del desarrollo nacional es la integración de la mujer a distintos ámbitos de la sociedad y la cultura y eso, posiblemente, tenga algún significado en sus vidas (Tarrés, 1997: 22).

El reto, entonces, queda planteado en la interrogante de cómo lograr el famoso equilibrio - el balance- entre lo mucho que queda por hacer incluida, por supuesto, la crítica hacia lo que se ha hecho mal, frente a los avances de diversa índole que indudablemente pueden documentarse en muchos estudios cuyo objetivo explícito o implícito es el análisis del cambio.

\section{Bibliografía citada}

Alexander, J., et al., 1987, The Micro-Macro Link, Berkeley University of California Press.

Davies, M., 1982, Woman's Place is at the Typewriter. Office Work and Office Workers, 1870-1930, Temple University Press, Philadelphia.

García, B., M. Blanco y E. Pacheco, 1999, "Género y trabajo extradoméstico”, en García, B. (coord.), Mujer, género y población en México, El Colegio de México y Sociedad Mexicana de Demografía, México.

INEGI/UNIFEM (Instituto Nacional de Estadística, Geografía e Informática/Fondo de Desarrollo de Naciones Unidas para la Mujer), 1995, La mujer mexicana: un balance estadístico al final del siglo XX, México.

Instituto Nacional de las Mujeres et al., 2001, El enfoque de género en la producción de las estadísticas sobre trabajo en México. Una guía para el uso y una referencia para la producción de información, México.

Knorr-Cetina, K. y A. Cicourel, 1981, Advances in Social Theory and Methodology. Toward an Integration of Micro and Macro-Sociologies, Routledge and Kegan, Boston.

Newman, I.y C. Benz, 1998, Qualitative-Quantitative Research Methodology. Exploring the Interactive Continuum, Southern Illinois University Press.

OIT (Organización Internacional del Trabajo), 2002, Informe sobre el empleo en el mundo, 2001. La vida en el trabajo en la economía de la información, Ginebra, Suiza.

Standing, G., 1999, Global Labour Flexibility. Seeking Distributive Justice, MacMillan Press Ltd., Great Britain; St. Martin's Press, Inc., Nueva York.

Tarrés, Ma. L. (comp.), 1997, La voluntad de ser. Mujeres en los noventa, PIEM de El Colegio de México.

Tashakkori, A. y C. Teddlie, 1998, Mixed Methodology. Combinig Qualitative and Quantitative Approaches, Applied Social Research Series, vol. 46, Sage Publications, Thousand Oaks, California. 